\section{P13 SYNTHETIC CONTROL METHODOLOGY AS A TOOL FOR EVALUATING PUBLIC HEALTH INTERVENTIONS - A LITERATURE REVIEW}

${ }^{1} \mathrm{~J}$ Bouttell ${ }^{*},{ }^{1} \mathrm{~J}$ Lewsey, ${ }^{2} \mathrm{~F}$ Popham, ${ }^{2} \mathrm{P}$ Craig, ${ }^{3} \mathrm{M}$ Robinson. ${ }^{1}$ Health Economics and Health Technology Assessment, University of Glasgow, Glasgow, UK; ${ }^{2} \mathrm{MRC/CSO}$ Social and Public Health Sciences Unit, University of Glasgow, Glasgow, UK; ${ }^{3}$ Public Health Science Directorate, NHS Health Scotland, Glasgow, UK

\subsection{6/jech-2018-SSMabstracts.139}

Background The synthetic control method (SCM) improves causal inference in non-randomised studies by building a counterfactual using a weighted combination of potential control units. Although it has been widely used in other disciplines it is not widely used in public health research. Our objectives were to identify the use of SCM studies in health and to summarise strengths and limitations identified in the literature. Methods We included studies that used a SCM design to investigate a health outcome of any intervention in any population. We searched for the term 'synthetic control method' in 26 health, social science and grey literature databases as well as checking for additional studies by key authors. No restrictions were placed on language or date. Searches were completed in February 2016. We summarised key information about the studies including setting, number of treated and control units, intervention and outcome, number of pre- and post-intervention data-points available and other methods used in the same study.

Results Searches identified 35 health-related studies of which 23 were from US settings and investigated a single treated unit. Most studies had at least 10 control units. Interventions investigated included health finance and health systems reform, industry regulation and taxation policies. Common outcomes were mortality rates and insurance rates/health care access. Most studies had more than 4 pre- and post-implementation data-points. SCM is most commonly used alongside differencein-difference methods. Advantages of SCM are that it does not rely on parallel pre-implementation trends and that it allows for time-varying unmeasured confounders. Limitations include the need for suitable data on both the treated unit and a pool of potential controls, difficulties if the treated unit is an outlier and the inapplicability of traditional statistical tests due to the small number of treated and control units and the fact that they have not been randomly allocated. Falsification tests are generally used as an alternative.

Conclusion This comprehensive literature review suggests thatSCM has been little used in health despite some advantages over existing methods. Future research incorporating the method, ideally in combination with other methods, would be of value.

\section{P14 A CRITICAL COMPARISON OF STATISTICAL AND INDIVIDUAL-BASED SIMULATION METHODS IN OBESITY RESEARCH}

${ }^{1,2}$ KF Arnold* ${ }^{*}, 2 \mathrm{MS}$ Gilthorpe, ${ }^{1,2}$ WJ Harrison, ${ }^{1,3} \mathrm{AJ}$ Heppenstall. ' Leeds Institute for Data Analytics, University of Leeds, Leeds, UK; ${ }^{2}$ School of Medicine, University of Leeds, Leeds, UK; ${ }^{3}$ School of Geography, University of Leeds, Leeds, UK

\subsection{6/jech-2018-SSMabstracts. 140}

Background It is widely acknowledged that the obesity epidemic exhibits many characteristics of a complex system individual heterogeneity and autonomy, interdependence, adaptivity, feedback loops, threshold effects, and emergence. Altering patterns of obesity requires robust methods that are capable of accurately estimating the causal effects of potential interventions in the presence of such complexity. Two groups of methods that have been proposed are of interest: (1) statistical regression models informed by directed acyclic graphs (DAGs), underpinned by graphical model theory; and (2) individual-based simulation models (IBSMs), consisting of microsimulation models (MSMs) and agent-based models (ABMs). Both groups of Methods may be used to evaluate causal effects as counterfactual contrasts, but methodological work seeking to compare and contrast the two methods is lacking, because they have only come to prominence relatively recently and have been largely confined to separate research disciplines. We sought to examine how these methods have been used within obesity research, identify the primary philosophical and methodological differences between them, and identify any implications for future causal analyses.

Methods We conducted searches of Medline, EMBASE, and Scopus to identify articles (from 1996-2016) that sought to make causal inferences relating to obesity, weight, or other closely associated health-related behaviours (e.g. exercise) by utilising either DAG-based statistical methods or IBSMs.

Results Our search returned 38 relevant articles utilising DAGbased statistical methods and 45 relevant articles utilising IBSMs (31 MSMs and $14 \mathrm{ABMs}$ ). From these results, we identified four primary differences between the two groups of Methods: (1) their relative reliance on theory versus data; (2) the timescales upon which they operate; (3) their relative focus on fixed versus random effects; and (4) the ways in which they assess the effects of (hypothetical) interventions.

Conclusion The results of our search cast light on how the two groups of methods might be used as complementary strategies, as well as the circumstances under which their causal Conclusions might diverge. DAG-based analyses, whilst mathematically robust, are not well-suited to scenarios in which data do not exist or are hard to measure, where the exposures/interventions of interest 'spillover' amongst individuals, and random effects are of specific interest. IBSMs, in contrast, are flexible enough to handle such complexity, though the robustness of their causal conclusions is in contention. Greater integration of the methods should be sought, as it has the potential to aid causal inferences in complex systems; to this end, further methodological work is needed.

\section{P15 LATENT CLASS REGRESSION MODELLING: A NOVEL APPROACH TO PREDICT SURVIVAL IN PATIENTS WITH CHRONIC HEART FAILURE (CHF)}

\footnotetext{
1,2,3 JL Mbotwa*, ${ }^{4} \mathrm{M}$ de Kamps, ${ }^{1,2} \mathrm{PD}$ Baxter, ${ }^{2} \mathrm{R}$ Cubbon, ${ }^{1,2} \mathrm{MS}$ Gilthorpe. 'Leeds Institute for Data Analytics, University of Leeds, Leeds, UK; ${ }^{2}$ School of Medicine, University of Leeds, Leeds, UK; ${ }^{3}$ Department of Applied Department, Malawi University of Science and Technology, Malawi; ${ }^{4}$ nstitute of Artificial and Biological Intelligence, School of Computing, University of Leeds, Leeds, UK
}

10.1136/jech-2018-SSMabstracts. 141

Background Chronic Heart Failure (CHF) is one of the leading cause of hospitalizations and deaths, more especially in old people, and this causes a substantial clinical and economic burden to the government. Using risk prediction models to accurately understand the dynamics of survival patterns amongst patients with CHF conditions would provide guidance to health care professionals in decision making on how 